Chapter 1

Are the Disk and Halo Axisymmetric? 


\title{
SHOULD THE OUTER MILKY WAY BE AXISYMMETRIC?
}

\author{
JAMES BINNEY \\ Department of Physics \\ 1 Keble Road, Oxford, OX1 3NP, England
}

\begin{abstract}
The shape of the outer Milky Way should be heavily influenced by the shape of our heavy halo. Simulations of the formation of collisionless halos indicate that they should be highly triaxial, with a tendency to prolateness. If our halo were as strongly triaxial as these simulations suggest, the outer disk would have ellipticity $\epsilon_{\text {disk }} \simeq 0.06$.

Simulations of galaxy formation which include an appropriately small fraction of gaseous matter give rise to halos of much smaller ellipticity, and predict $\epsilon_{\text {disk }} \lesssim 0.02$. These simulations are not as reliable as purely collisionless simulations, and we should treat their results with some caution. However, it is not incredible that a small fraction of gas could radically reduce the ellipticity of a halo by making the system's potential more centrally concentrated.

The distribution of the apparent ellipticities of spiral galaxies indicates that their disks have slightly eliptical disks, $\epsilon_{\text {disk }} \simeq 0.05$. This may merely reflect ephemeral features such as spiral arms, however. The velocity fields of disks provide a more robust probe of the ellipticity $\epsilon_{\Phi}$ of galaxy potentials. The narrowness of the Tully-Fisher relation places an interesting upper limit $\epsilon_{\Phi}<0.1$. More detailed studies of individual velocity fields will be required if we are unambiguously to demonstrate $\epsilon_{\Phi}>0$ in some cases.
\end{abstract}

\section{Introduction}

It is now generally accepted that elliptical galaxies are not axisymmetric and it is natural to wonder whether the same might be true of disk galaxies such as the Milky Way. Of course, at least a half of all disk galaxies are clearly non-axisymmetric at small radii because they contain a bar. There is steadily accumulating evidence that the Milky Way has a central bar too. 
But the dynamical effects of this bar would be very small at the solar radius, $R_{0}$, and beyond. What I want to discuss here is the possibility that the outer galaxy is not so much barred as mildly elliptical. The main motivation for this proposition is theoretical and connected with the existence of massive halos. In Section 2 I review these arguments. Since these are of a general nature and would apply to any disk galaxy, it is natural first to test them by examining in Section 3 the evidence for ellipticity in external disk galaxies. Weinberg (this volume) examines the evidence for and against the Milky Way's being elliptical.

\section{Theoretical arguments}

Until recently it has been assumed that disk galaxies are fundamentally round rather than elliptical in shape, even though their images are usually elliptical - by Ockham's razor the ellipticity of their images was entirely ascribed to the effects of projection rather than to some combination of intrinsic ellipticity and projection.

From the mid 1970s this traditional view has been undermined by the suspicion that disk galaxies are embedded in dark halos (Ostriker \& Peebles 1973, Trimble 1987). The Milky Way's dark halo is reputed to contribute on the order of half the galactocentric force on the Sun, and to contain the overwhelming majority of the Milky Way's total mass (Fich \& Tremaine 1991). In 1978 Binney pointed out that it would be natural for dark halos to be triaxial bodies, independently of how they formed or of what they are made, and investigated the consequences of this triaxiality for the embedded visible galaxies.

The argument for the triaxiality of halos is simple. These systems (unlike disks) probably formed collisionlessly. Numerical experiments show that the 'violent' relaxation that takes place during the virialization of a collisionless system, is never so efficient as completely to erase memory of the initial conditions. In any plausible scenario of galaxy formation, the latter will have been significantly anisotropic, and the numerical experiments (Binney 1976, Aarseth \& Binney 1978) indicated that this anisotropy will have bequeathed to the final halo a triaxial form.

Over the last decade and a half the sophistication of the numerical experiments has increased enormously, and we have achieved a moderate understanding of the internal dynamics of triaxial systems, although the topic is still very much under study.

The greatest advance in the numerical experiments has been the simulation of galaxy formation in realistic cosmological contexts and the inclusion, in a crude way, of gas. Barnes (1992, 1994), Hernquist (1993) and others have studied mergers in small groups of galaxies, Frenk et al. (1988), 
Dubinski \& Carlberg (1991) and Warren et al. (1992) have studied the formation of galaxy halos through hierarchical clustering in large cosmological simulations, and Katz \& Gunn (1991) have studied the formation of individual galaxies from lumpy protoclouds along the lines pioneered by van Albada (1982). The purely collisionless simulations confirm Binney's original contention that halos will be generically triaxial - they prove strongly triaxial with a tendency to prolateness rather than oblateness. Moreover, the structures of merger remnants frequently deviate from axisymmetry in more exotic ways than mere triaxiality (Barnes 1992).

Simulations that contain even a little mass in 'gas' particles yield significantly different results (Katz \& Gunn 1991, Barnes 1994). Collisional particles rapidly sink to the centres of merger remnants, greatly increasing the density there. This seems to drive the dark halo away from prolateness towards oblateness. For example, Barnes (1994) describes two merger simulations that initially differed only in that $1.5 \%$ of the mass of one simulation was gaseous. In the gas-free simulation the innermost quartile of the dark halo ended with axis ratios 1:0.88:0.80, while in the gas-rich simulation the inner dark halo finished with axis ratios 1:0.96:0.87 (Barnes, private communication). Thus $1.5 \%$ of gas reduced the ellipticity in the equatorial plane by a factor of $\sim 3$.

\subsection{SELF-GRAVITY OF THE DISK}

What makes the ellipticity $\epsilon_{h}$ of dark halos sensitive to the inclusion of so little gas? One factor to consider, which is certainly an important one in the Milky Way, is the self-gravity of the disk. Given that the pattern speed of the halo's figure must be very slow, it is straightforward to derive two results from the epicycle approximation: (i) the nearly closed orbits of the disk align such that their major axes coincide with the minor axis of the halo; (ii) the ellipticity $\epsilon_{d}$ of these orbits is nearly equal to that of the ellipticity $\epsilon_{\Phi}$ of the isopotential contours. The ellipticities of disk and the closed orbits are strictly different but at the radii of interest very similar: for an exponential disk of scale-length $R_{d}, \epsilon_{\text {disk }} \simeq\left(1+R / R_{d}\right) \epsilon_{d}$ (Franx \& de Zeeuw 1992). I shall identify $\epsilon_{d}$ with $\epsilon_{\text {disk }}$.

Since it is characteristic of solutions of Poisson's equation that $\epsilon_{\Phi}$ is roughly a third of the ellipticity of the generating mass distribution, we may write

$$
\epsilon_{\Phi} \simeq \frac{1}{3}\left(\frac{M_{h}}{M_{h}+M_{d}} \epsilon_{h}-\frac{M_{d}}{M_{h}+M_{d}} \epsilon_{d}\right)
$$

where $M_{h}$ and $M_{d}$ are the masses of the halo and disk interior to the radius 
under consideration. Inserting $\epsilon_{d} \simeq \epsilon_{\Phi}$ into this relation, we find

$$
\epsilon_{d} \simeq \epsilon_{\Phi} \simeq \frac{M_{h}}{3 M_{h}+4 M_{d}} \epsilon_{h} .
$$

Near the Sun, $M_{h} \simeq M_{d}$ and $\epsilon_{d} \simeq \frac{1}{7} \epsilon_{h}$, while at radii so large that $M_{h} \gg$ $M_{d}, \epsilon_{d} \simeq \frac{1}{3} \epsilon_{h}$. In other words, near $R_{0}$ the self-gravity of the disk diminishes the ellipticity of the potential by a factor $\frac{3}{7}$. Moreover, this is an underestimate of the true diminution, because our simple calculation has not allowed for the fact that the halo will be made rounder by this rounding out of the potential. Barnes' pair of simulations with and without gas may reflect this effect. In any event, taking the mean ellipticity $1-b / a \simeq 0.4$ of the halos studied by Dubinski (1992) and one third of this value $\epsilon_{h}=0.13$, as typical for simulations with and without gas, we find $\epsilon_{d}=0.04,0.13$ in the case $M_{h} \gg M_{d}$ discussed by Binney (1978) and $\epsilon_{d}=0.019,0.06$ in the more realistic case $M_{h} \simeq M_{d}$.

It is, in fact, likely that for a sufficiently large value of $M_{d} / M_{h}$ both disk and halo become perfectly round. It would be interesting to determine this value of $M_{d} / M_{h}$.

\subsection{CENTRALLY CONCENTRATED SYSTEMS}

The effect of the disk's self-gravity just described will be small outside a few effective radii, $R_{e}$. Yet Dubinski (1994) has shown that adding the potential of a luminous galaxy to a dark halo can diminish the halo's ellipticity at several $R_{e}$. In his experiments an analytic disk or bulge potential placed at the centre of an $n$-body halo has a slowly increasing mass. While the flattening $c / a$ of the halo is only slightly reduced by the growth of the analytic potential, the halo's axis ratio $b / a$ increases from $b / a \simeq 0.6$ to $b / a \simeq 0.8$ at $r \simeq 25 \mathrm{kpc}=3.1 R_{e}$, and from from $b / a=0.7$ to $b / a=0.85$ as far out as $r=40 \mathrm{kpc}=5 R_{e}$. The analytic potential has these far-reaching effects because the orbital structure of a triaxial system depends qualitatively on the system's degree of central concentration. This phenomenon deserves close attention because real galactic potentials are more centrally concentrated than that of any $n$-body model.

The landmarks in our understanding of the internal dynamics of triaxial systems are Schwarzschild's (1979) analysis of the orbit mix of a typical triaxial system, and de Zeeuw's (1985) demonstration that Stäckel potentials provide analytic models of the orbits identified by Schwarzschild. Wilkinson \& James (1982), Barnes (1992) and others have demonstrated that the bars formed in collisionless $n$-body experiments conform to the pattern established by Schwarzschild and de Zeeuw. In particular, the backbones of these systems comprise stars on 'box' orbits. 
The conversion of even a small fraction of the total mass to collisional 'gas' particles radically changes the structure of merger remnants because the great increase in the final central density to which these particles give rise, radically restructures parts of phase space. No analytic counterparts are known for the orbits in a centrally concentrated potential which take over the part of phase space which is occupied by box orbits in a Stäckel potential. Consequently, the dynamics of these crucial orbits is not properly understood.

For want of a better framework, one usually discusses the dynamics of centrally concentrated triaxial potentials by reference to Stäckel potentials. In this perhaps inappropriate language, as an initially Stäckel-like potential is made more centrally concentrated, more and more box orbits become either trapped by resonances, or become stochastic through competition between resonances for their adherence (Gerhard \& Binney 1985, Lees \& Schwarzschild 1992, Schwarzschild 1993). Physically, what is happening here is that orbits that pass close by the centre are scattered by the strong gravitational field there and are classified as stochastic orbits. Meanwhile other orbits perpetually avoid the centre by coordinating their motions parallel to the potential's principal axes; that is, by being trapped by some resonance.

Schwarzschild (1993) determined the orbital structure of the potential generated by the density distribution $\rho \propto m^{-2}$, where $m^{2}=x^{2} / a^{2}+y^{2} / b^{2}+$ $z^{2} / c^{2}$ is an elliptical radius. He showed that in this scale-free limit no nonresonant regular box orbits survive. He was nevertheless able to reproduce the underlying density distribution by superposing orbits in the given potential, provided he employed stochastic as well as regular orbits. This exercise suggests that realistically centrally concentrated triaxial halos may be possible, but it does not demonstrate that they will form in the real universe.

There are two potential problems with the orbit distribution of Schwarzschild's self-consistent scale-free model: it may be too special to form during violent relaxation or merging; or it may not be the distribution that emerges as dissipation makes a Stäckel-like system more centrally concentrated. I think it will be hard convincingly to eliminate these two anxieties because the enormous range in time- and length-scales of realistically centrally concentrated systems makes them difficult to simulate accurately. On the other hand, the approach pioneered by Schwarzschild cannot be used to study how slowly an initially triaxial system evolves towards axisymmetry in the event that a strict triaxial equilibrium is impossible. My hunch is that at the end of the day it will be found that centrally concentrated triaxial halos do form and survive for much longer than the Hubble time. I base this hunch on two facts. (i) Gerhard \& Binney (1985) estimated the 
time required for the triaxiality of an elliptical galaxy to disappear as a consequence of stars being scattered off the all important box orbits by a nuclear cusp. They concluded that in the Hubble time this process would only destroy triaxiality inside $\sim 3 \mathrm{kpc}$. (ii) The central concentration of the potential is important only for stars whose apocentres lie many core radii out. But these are the stars which are most sensitive to any figure rotation of the potential; if the potential has non-zero pattern speed, as it surely has, the Coriolis force will deflect them from the nucleus, with the consequence that they are never scattered by it.

Before turning from dynamics to phenomenology, I must make explicit the spectre implicit in the discussion above. There must be a largest radius $r_{\max }$ at which a galaxy may safely be treated as a relaxed quasi-equlibrium object that may be plausibly characterised by a pair of ellipticities. Beyond $r_{\max }$ the system will be lop-sided and irregularly time-dependent as a consequence of long local dynamical times and regular passages by lumps of material (mostly dark) moving on highly elliptical orbits. We have no reliable estimate of $r_{\max }$. A lower estimate would be the radius, $r_{\text {warp }} \simeq 1.2 R_{0}$, at which warps become pronounced. In the popular normal-mode theory of warps (Dekel \& Shlosman 1983, Toomre 1983), $r_{\text {warp }}$ would be an underestimate of $r_{\max }$, but as Kuijken \& Tremaine (1994) have pointed out, the Milky Way's warp is significantly too asymmetric to be accounted for by the normal-mode theory, and we should seriously consider the possibility that $r_{\max } \simeq r_{\text {warp }}$ as an alternative theory (Ostriker \& Binney 1989) requires. Beyond $r_{\max }$ the disk will not merely be elliptical, but lop-sided as well. Richter \& Sancisi (1994) have recently demonstrated that most disk galaxies are significantly lop-sided at a few $R_{e}$.

\section{Are the disks of external galaxies elliptical?}

If disk galaxies are triaxial rather than axisymmetric, one would expect the principal axes of their isophotes to 'twist' from one isophote to the next. Bertola, Vietri \& Zeilinger (1991) have sought such isophote twists in a sample of 32 nearby spirals. They find that the majority of these galaxies display isophote twists, from which it follows that in these galaxies at least one of the bulge and the disk must be triaxial.

There are two obvious observational tests of the conjecture that galactic disks are circular. One is to examine the distribution of apparent ellipticities. When a flat disk is viewed in projection, its image may have any axis ratio $q_{\text {app }}$ from 1 (circular) to 0 (linear). If the disk is circular, the probability distribution $P\left(q_{\mathrm{app}}\right)$ is independent of $q_{\mathrm{app}}$, but if the disk has intrinsic axial ratio $q_{\text {true }}<1, P\left(q_{\text {app }}\right)$ peaks at $q_{\text {app }}=q_{\text {true }}$ and falls away quite steeply at larger values of $q_{\text {app }}$. This has the effect of reducing the number 
of apparently round galaxies one expects to find if a significant fraction of disks are intrinsically elliptical. Binney \& de Vaucouleurs (1981) found marginal evidence for this effect in the 650 spirals in second Reference Catalogue of Galaxies, while Lambas, Maddox and Loveday (1992) detected it in the 13500 spiral galaxies in the APM survey. The data favour many disks having axis ratio $q_{\text {true }} \simeq 0.95$, with few disks more elliptical than $q_{\text {true }}=0.9$. Huizinga \& van Albada (1992) and Franx \& de Zeeuw (1992) have reached similar conclusions.

The apparent ellipticities of spiral galaxies must be influenced by spiral structure, so it is not clear whether the results reported in the last paragraph reflect fundamental ellipticity or the ephemeral effects of spiral arms. A more robust test of the conjecture that disks are intrinsically round is to examine the velocity fields of galaxies (Binney 1978, Franx \& de Zeeuw 1992, Franx, van Gorkom \& de Zeeuw 1994). The kinematic minor axis is the line on which the projected velocity equals the systemic velocity, while the kinematic major axis is the line along which the projected rotation velocity is largest for a fixed galactocentric distance. Since the stars of an elliptical disk are on elliptical rather than circular orbits, the kinematic axes usually do not coincide with the photometric principal axes when the velocity field generated by stars on elliptical orbits is examined in projection. Unfortunately, warping of the disk also causes the kinematic axes to deviate from the photometric axes. The effects of warping and ellipticity can in principle be distinguished because the former keeps the kinematic axes mutually perpendicular, while the latter does not. However, separating warps from ellipticity requires detailed modelling of the data, and the sample of galaxies that have been analyzed in the requisite detail is still small. Begeman (1989) studied eight galaxies in detail and found misalignments of the kinematic and photometric axes compatible with those expected if the galaxies had potentials of ellipticity $\epsilon_{\Phi} \simeq 0.1$, but the offsets detected were comparable to the errors. Franx et al. (1994) have analyzed HI data for a filled ring at $R \simeq 6.5 R_{e}$ around an early-type galaxy in Fornax cluster, IC 2006. Although the circular-speed curve appears to be roughly flat to this radius, Franx et al. find that the potential of IC 2006 is axisymmetric to within the errors: $\epsilon_{\Phi}=0.012 \pm 0.026$. On the other hand dark halos do not as a rule prove to be spherical: Sackett et al. (1994) deduce from observations of a polar ring that the halo of NGC $4650 \mathrm{~A}$ is as flattened as the luminous galaxy.

Franx \& de Zeeuw (1992) pointed out that disk ellipticity would contribute to the scatter in the Tully-Fisher relation, $L \propto v_{c}^{n}$, between luminosity and maximum circular speed $v_{c}$, by making the inferred value of $v_{c}$ dependent on the orientation of the disk. The magnitude of this effect depends on how $v_{c}$ is inferred from the data - whether the disk's orientation 
is determined by fitting a ring model to the velocity field, or by fitting an axisymmetric model to the photometry; a ring model yields the smaller error. The magnitude of the error also depends on the inclination angle $i-$ the error in $v_{c}$ becomes large for nearly face-on galaxies. Franx \& de Zeeuw calculated the ellipticity $\epsilon_{\Phi}$ that would give rise to a scatter in $L \propto v_{c}^{n}$ as large as that reported, and found that $\epsilon_{\Phi} \simeq 0.1$ sufficed because even the small errors in $v_{c}(10 \%)$ give rise to significant scatter in $L(\simeq 40 \%)$. Since it seems unlikely that disk ellipticity is the only contributor to the scatter in $L \propto v_{c}^{n}$, Franx \& de Zeeuw conclude that $\epsilon_{\Phi}<0.1$.

\section{Conclusion}

The shape of outer Milky Way will be heavily influenced by the shape of the heavy halo. $N$-body simulations of the formation of collisionless halos indicate that such halos would be strongly triaxial, and the dynamics that underlies this result is understood in some detail. Disk ellipticities of order $\epsilon_{\text {disk }} \simeq 0.06$ are predicted by these simulations for radii near $R_{0}$ (where half of the radial force is contributed by the disk) rising to $\epsilon_{\text {disk }} \simeq 0.13$ at $R \gg R_{0}$.

The halos that form in galaxy-formation simulations that include gas are significantly rounder than those that form in purely collisionless simulations. These still pioneering simulations are undoubtedly less trustworthy than are purely collisionless simulations, but we do expect the inclusion of gas to reduce significantly the ellipticities of halos. One mechanism for this reduction is the tendency of disks to anti-align with the potential in which they move, thus diminishing the ellipticity of both the overall potential and the halo. A second more far-reaching mechanism arises from the fact that orbits in very centrally concentrated triaxial potentials are not structured in the same way as orbits in less centrally concentrated systems. Because of this, very centrally concentrated self-consistent triaxial systems may not even exist.

Notwithstanding these anxieties, our best guess at the moment is that halo potentials are at least mildly triaxial, with the consequence that the embedded disks will have ellipticities $\epsilon_{\text {disk }} \simeq 0.015-0.03$, with larger values ruling at larger radii. Studies of the apparent-ellipticity distribution of spiral galaxies suggest that most disks are elliptical, with ellipticities near 0.05 . In principle the velocity fields of disks provide much more reliable probes of disk ellipticity than photometry, and this line of attack should be increasingly exploited in the future as good quality velocity data become available. At the moment is hard to draw a firm conclusion from detailed studies of individual data, although these do seem to be compatible with an ellipticity distribution centred on $q=0.05$ of width $\delta \epsilon \simeq 0.05$. Signifi- 
cantly more elliptical disks seem to be ruled out by the narrowness of the Tully-Fisher relation.

\section{References}

Aarseth, S.J., Binney J.J., 1978. MNRAS, 185, 227

Barnes, J.E., 1992. ApJ, 393, 484

Barnes, J.E., 1994. In The Formation of Galaxies, ed. Munoz-Tunon, C., (Cambridge, Cambridge University Press)

Begeman, K., 1989. A\&A, 223, 47

Bertola, F., Vietri, M., Zeilinger, W.W., 1991. ApJ, 374, L13

Binney, J.J., 1976. MNRAS, 177, 19

Binney, J.J., 1978. MNRAS, 183, 779

Binney, J.J., de Vaucouleurs, G., 1981. MNRAS, 194, 679

Dekel, A., Shlosman, I., 1983. In IAU Symposium 100, Internal kinematics \& dynamics of galaxies ed. Athanassoula, E., pp. 187-188. Dordrecht: Reidel

de Zeeuw, P.T., 1985. MNRAS, 216, 273

Dubinski, J., 1992. ApJ, 401, 441

Dubinski, J., 1994. ApJ, 431, 617

Dubinski, J., Carlberg, R.G., 1991. ApJ, 378, 496

Fich, M., Tremaine, S., 1991. ARA\&A, 29, 409

Franx, M., de Zeeuw, P.T., 1992. ApJ, 392, L47

Franx, M., van Gorkom, J.H., \& de Zeeuw, P.T., 1994. ApJ, 000, 000

Frenk, C.S., White, S.D.M., Davis, M., Efstathiou, G., 1988. ApJ, 327, 507

Gerhard, O.E., Binney, J.J., 1985. MNRAS, 216, 467

Hernquist, L., 1993. ApJ, 409, 548

Huizinga, J.E., van Albada, T.S., 1992. MNRAS, 254, 677

Katz, N., Gunn, J.E., 1991. ApJ, 377, 365

Kuijken, K., Tremaine, S.D., 1994. ApJ, 421, 178

Lambas, D.G., Maddox, S.J., Loveday, J., 1992. MNRAS, 258, 404

Lees, J., Schwarzschild, M., 1992. ApJ, 384, 491

Ostriker, E.C., Binney, J.J., 1989. MNRAS, 237, 785

Ostriker, J.P., Peebles, P.J.E., 1973. ApJ, 186, 467

Richter, O.-G., Sancisi, R., 1994. A\&A, 000, 000

Sackett, P.D., Rix, H-W., Jarvis, B.J., Freeman, K.C., 1994. ApJ, 000, 000

Schwarzschild, M., 1979. ApJ, 232, 236

Schwarzschild, M., 1993. ApJ, 409, 563

Toomre, A., 1983. In IAU Symposium 100, Internal kinematics $\&$ dynamics of galaxies ed. Athanassoula, E., pp. 177-186. Dordrecht: Reidel

Trimble, V., 1987. ARA\&A, 25, 425

van Albada, T.S., 1982. MNRAS, 201, 939

Warren, M.S., Quinn, P.J., Salmon, J.K., Zurek, W.H., 1992. ApJ, 399, 405

Wilkinson, A., James, R.A., 1982. MNRAS, 199, 171

\section{DISCUSSION}

K. Freeman: Early in your talk, you suggested that the large size of the halo would limit its possible pattern speed. Could you say more about such limits on the halo pattern speed, please?

Binney: One cannot speak confidently of a perimeter of something one 
cannot see. Bars in N-body models end by co-rotation at the latest. So the simple argument is to say $\omega_{p}<V_{c} / R_{\text {halo }}$ where $\mathrm{R}_{\text {halo }}>50 \mathrm{kpc}$ at least. Logically this upper limit could be too small because the halo could have a fast rotating subsystem inside it, and be axisymmetric beyond the boundary of the subsystem. But N-body models give no indication that halos, which being dissipationless cannot concentrate their angular momentum, are structured like that. 